\title{
20 MHZ, SUB-PS, TUNABLE TI:SAPPHIRE LASER SYSTEM FOR REAL TIME, STAIN FREE, IN VIVO HISTOLOGY OF THE SKIN
}

\author{
Róbert Szipőcs $^{1,2}$, Luca Fésüs ${ }^{1,3}$, Ádám Krolopp ${ }^{1,2}$, Ernő Hettinger ${ }^{1,2}$, Lajos Vass $^{2}$, Norbert

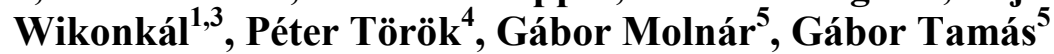 \\ ${ }^{I}$ Wigner RCP, Institute for Solid State Physics and Optics, P.O. Box 49, H-1525 Budapest, Hungary \\ ${ }^{2} R \& D$ Ultrafast Lasers Ltd, Konkoly-Thege str 29-33, H-1121 Budapest, Hungary \\ ${ }^{3}$ Department of Dermatology, Venereology and Dermatooncology, Semmelweis University, Budapest, Hungary \\ ${ }^{4}$ Nanyang Technological University, Environmental Life Sciences Engineering, Singapore 639798, Singapore \\ ${ }^{5}$ MTA-SZTE Research Group for Cortical Microcircuits, University of Szeged, Szeged, H-6726, Hungary
}

DOI: https://doi.org/10.14232/kvantumelektronika.9.33

\section{Introduction}

Coherent anti-stokes Raman scattering (CARS) [1] microscopy is widely used in label-free biomedical imaging applications. For in vivo diagnostic use of CARS microscopy, wide field detection is preferred to descanned configurations [2]. Chemical selectivity poses a major difficulty when femtosecond (fs) pulse lasers are applied, as their spectral bandwidth is typically significantly higher $(\sim 5-10 \mathrm{~nm})$ than the optimum value $(\sim 1 \mathrm{~nm})$ matching the bandwidth of molecular vibrations. This fact leads to the appearance of an enhanced non-specific background and the decrease of spectral sensitivity in CARS imaging. Two years ago we proposed a fast spectral modulation technique for sub-100 fs pulse Ti:S lasers [3], which allowed us to modulate the laser spectrum on ms time scale with the use of a piezo-driven Michelson interferometer. In one of the settings we used, we modulated the laser spectrum of our laser in such a way, that CARS imaging for $\mathrm{CH}_{2}$ bonds in "lipids" and $\mathrm{CH}_{3}$ bonds "proteins" did not require any tuning of the pump (Ti:S) laser or any readjustment of the delay between the pump and Stokes (Yb-amplifier) pulses, which allowed us to record stain-free histological images [4] of brain slices. In this paper we report on a newly developed, $20 \mathrm{MHz}$, sub-ps Ti:sapphire laser system, which supports real time, in vivo, twochannel, high chemical contrast, DVRF CARS imaging, i.e. histology of the skin by a commercial LSM 7 MP microscope (Carl Zeiss, Jena, DE) without any modification of its ZEN software or post-processing of the images like in case of our previous CARS setups used for histology $[3,4]$.

\section{Experimental setup}

For our comparative studies, we used two different CARS imaging setups, as shown in Fig.1. In our setup at the University Szeged (USZ) [3,5], we used a $\sim 80 \mathrm{MHz}, \sim 80$ fs Ti:S laser (Mai Tai, Newport Spectra-Physics, USA) as a pump laser (for details, see Refs. 3 and 5). In the setup at Wigner RCP, Budapest [1,4], we replaced our $\sim 76 \mathrm{MHz}, \sim 150$ fs Ti:S laser by a newly developed, $\sim 20 \mathrm{MHz}$ repetition rate, sub-ps Ti:S laser (FemtoRose TUN LC GTI, R\&D Ultrafast Lasers Ltd.). The long cavity laser configuration was similar to that was published in Ref. 6, with a few modifications, among them the most critical was the following: we replaced the SF10 prism pair by a Gires-Tournois interferometer, which provided considerably higher intracavity dispersion than the prism pair previously used. Beside a birefringent tuning element, fine tuning of the Ti:sapphire laser was obtained by the piezo controlled GTI. In our new setup, the spectral bandwidth of the pump (Ti:S) laser was reduced from 6-8 $\mathrm{nm}$ to $\sim 1-2 \mathrm{~nm}$. Accordingly, the pulse duration increased from $\sim 150$ fs to $\sim 600 \mathrm{fs}$, or slightly above. This four-fold reduction in the peak intensity was compensated by the lower repetition rate of our long cavity Ti:sapphire laser comprising a Herriottcell and a $\sim 2 \mathrm{~W}$ average power, $532 \mathrm{~nm}$ pump laser [6]. Pulse duration of the $\sim 20 \mathrm{MHz}$ laser was characterized by a PulseCheck autocorrelator (APE GmbH, DE). Depending on the intracavity 
dispersion set by the mirror spacing of an intracavity GTI, the pulse duration could be set in the 0.6$1 \mathrm{ps}$ range. Spectral bandwidth of the $\sim 20 \mathrm{MHz}$ Ti:sapphire laser (pump) was measured $\Delta \lambda<2 \mathrm{~nm}$ allowing high spectral resolution DVRF-CARS imaging. For higher spectral contrast between the anti-Stokes signals generated by „lipids” and „proteins”, we placed a Michelson interferometer similar to that was used in Ref. 3 into the beam path of our Stokes (Yb) laser. By spectral modulation, we obtained a double peaked spectrum with a peak separation of 5-6 nm at around $1030 \mathrm{~nm}$. DVRF CARS imaging was performed by two NDD detectors of our microscope: the antiStokes signals for „lipids” and ,proteins” were separated by a dichroic mirror with a long pass edge at around $645 \mathrm{~nm}$, while two bandpass filters with central wavelengths at $641 \mathrm{~nm}$ and $650 \mathrm{~nm}$ were respectively placed in front of the NDD-s. The optical signal detected by the „lipid" detector was pseudo-colored red, while that of the ,protein” was given the color blue to match conventional H\&E stained histology.
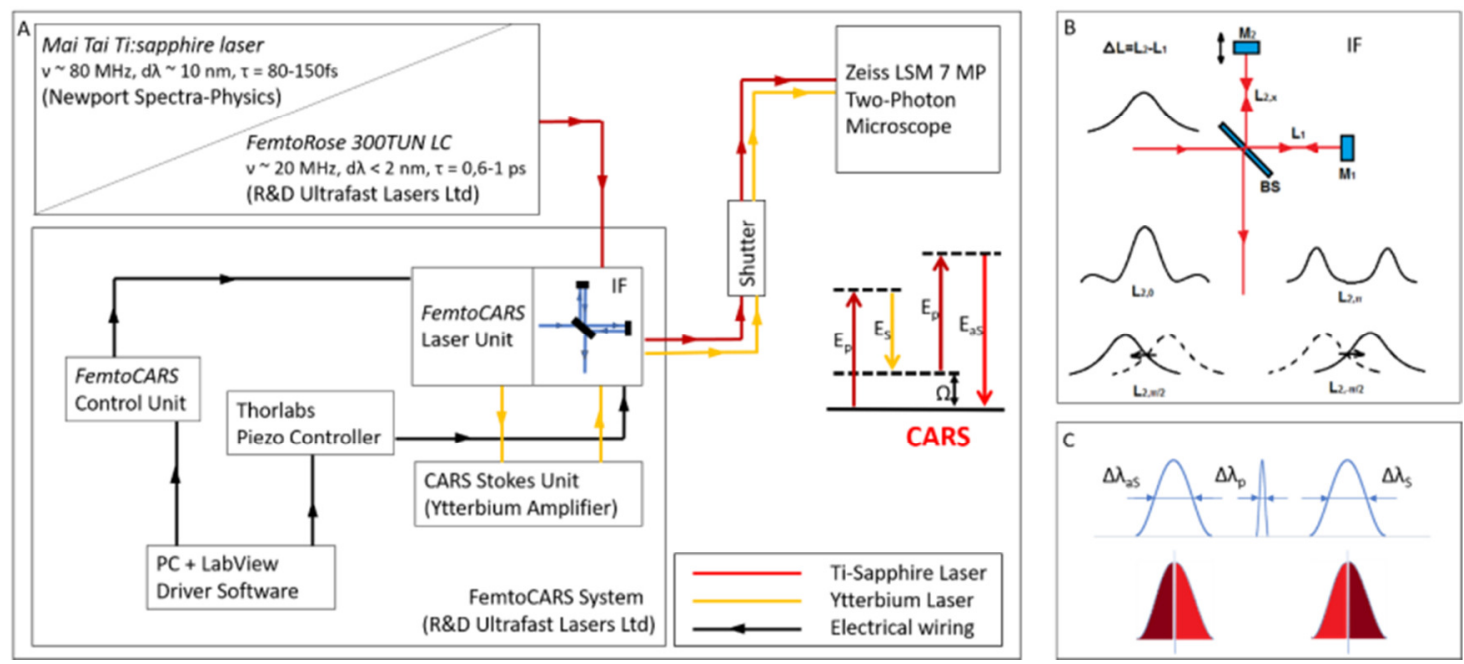

Figure 1

(A) Layout of the experimental setups used for IF-CARS [3] and real time DVRF CARS imaging with our newly developed, 20 $\mathrm{MHz}$, sub-ps Ti:S laser. In both setups, a Michelson-interferometer is used for spectral modulation of either the pump (IF-CARS setup, [3]) or Stokes pulses (real time DVRF CARS setup). By electonically modulating the phase difference $(\Delta \varphi)$ of the two mirrors, different output spectra can be obtained. B) Phase difference $\Delta \varphi=0$ at the central frequency results in a narrower spectrum, whereas $\Delta \varphi=\pi$ results in a double-peaked spectrum around the central wavelenght, when the path difference offset, $\Delta L$ is properly set. When the phase difference is $\pi / 2$ or $-\pi / 2$, two different spectrally shifted laser spectra is obtained with two maxima a few nm-s apart [3]. C) For high chemical contrast, real time DVRF-CARS imaging with two parallel NDD detectors, at least one of the pump or Stokes laser spectral bandwidth has to be small enough (<2 $\mathrm{nm}$ ) to create properly distinguishable anti-Stokes signals. If $\Delta \lambda_{p}$ is small $\left(\sim 2 \mathrm{~nm}\right.$ ) and $\Delta \lambda_{S}$ is broader ( $10 \mathrm{~nm}$ ), nearly half of the Stokes photons (of lower energy) excites the $\mathrm{CH}_{2}$ bonds in lipids, while others (of higher energy) excite the $\mathrm{CH}_{3}$-bonds in proteins.

\section{Results}

Histological imaging experiments on ex vivo human and murine skin samples by different CARS imaging methods are summarized in Fig. 2 and Fig. 3. In Fig 2A, a "quasi-H\&E color encoded", composite, CARS image is shown (after post-processing), for which two CARS images were recorded for ,protein” and „lipid” settings in human basal cell cancer (BCC). For recording of these, a Mai Tai pump laser was respectively tuned to 790 and 798 nm [4]. In Fig. 2B), an IF-CARS histological image of murine skin (after post-processing) is shown, for which a spectrally modulated Mai Tai pump laser was used for recording "protein" and "lipid" CARS images with optical pulses with spectral maxima at 792 and $796 \mathrm{~nm}$, respectively [3]. 

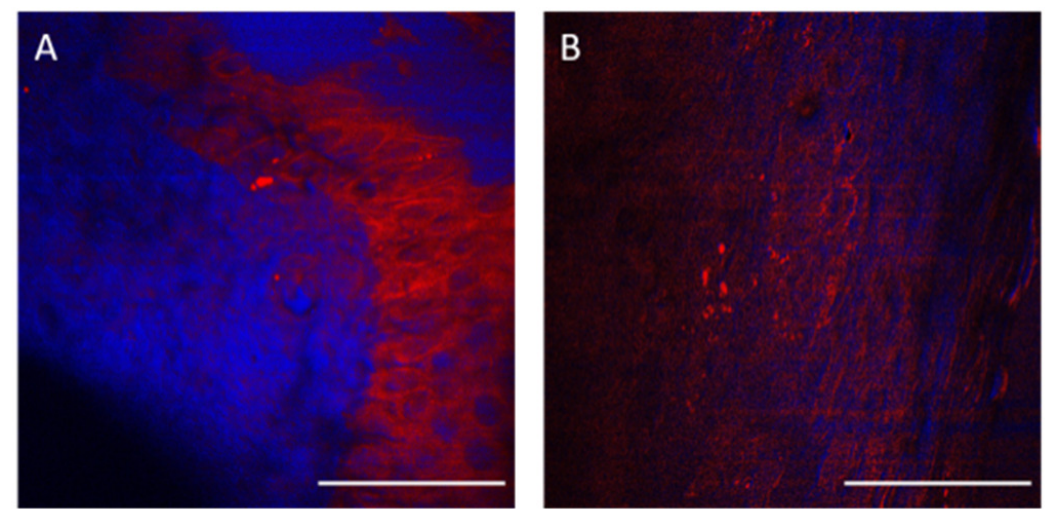

Figure 2

Histological imaging of ex vivo human and murine skin samples by different CARS imaging methods. A) Composite of CARS images recorded for "proteins" and "lipids" in human basal cell cancer (after post-processing) with a Mai Tai pump laser tuned to 790 and $798 \mathrm{~nm}$, respectively [4]. Time required for recording and processing: c.a. $5 \mathrm{~min}$. B) IF-CARS imaging of murine skin (after post-processing) with spectrally modulated Mai Tai pump laser pulses with spectral maxima az 792 and $796 \mathrm{~nm}$ [3]. Time required for recording and processing: c.a. $5 \mathrm{sec}$. Scalebar on both figures: $50 \mu \mathrm{m}$

In Fig. 3, we compare stain free histological images of skin samples by parallel, two-channel detection of "protein" and "lipid" CARS signals, referred to as DVRF CARS, for which we used a i.) $\sim 80 \mathrm{MHz}$ repetition rate, $\sim 80 \mathrm{fs}(\Delta \lambda \sim 10 \mathrm{~nm}$ ) Mai Tai pump laser tuned to $796 \mathrm{~nm}$ (Fig. 3A), and ii.) our newly developed, $\sim 20 \mathrm{MHz}$ repetition rate, $\sim 0.8 \mathrm{ps}(\Delta \lambda \sim 1 \mathrm{~nm})$ FemtoRose TUN LC GTI pump laser tuned to $794 \mathrm{~nm}$ together with a Yb-fiber laser having a double-peaked (off resonance, see Fig. 1b ) spectrum.
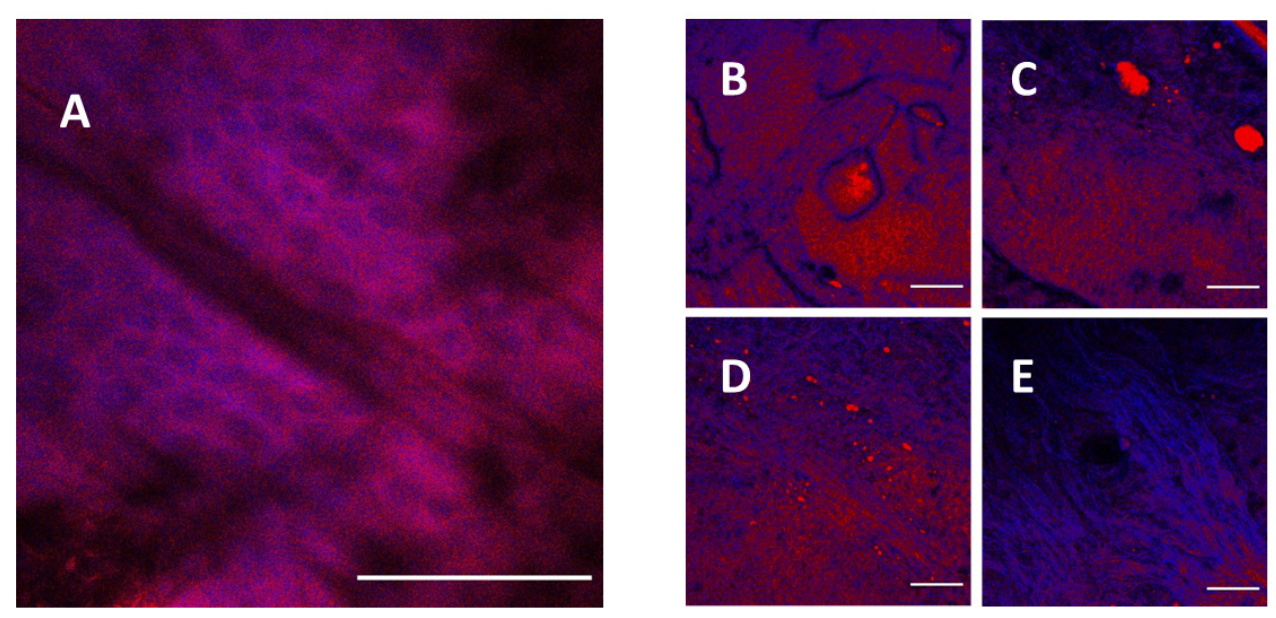

Figure 3

Stain free histological imaging of skin samples by DVRF CARS A.) Post-processed DVRF-CARS image of an ex vivo murine skin sample using a $\sim 80 \mathrm{MHz}$ repetition rate, $\sim 80 \mathrm{fs}(\Delta \lambda \sim 10 \mathrm{~nm})$ Mai Tai pump laser tuned to $796 \mathrm{~nm}$ with simultaneous detection of the two anti-Stokes signals. Time required for recording and processing: c.a. 1 sec. B-E) Real time, DVRF-CARS images of ex vivo human skin with simultaneous detection of the lipid (,,- $\mathrm{CH}_{2}$ vibration") and protein (,,- $\mathrm{CH}_{3}$ vibration”) channels with a 20 $\mathrm{MHz}$ repetition rate, $0.8 \mathrm{ps}(\Delta \lambda \sim 1 \mathrm{~nm})$ FemtoRose TUN LC GTI pump laser tuned to $794 \mathrm{~nm}$ and with a double-peaked (off resonance, see Fig. 1b) Yb-laser spectrum. Time required for recording and processing: c.a. 0.1-1 sec. Nuclei and hair appear in blue, cytoplasm and cell membrane in red. Scalebar on each figure: 50 mm. Resolution in each Figure: 512x512 pixels. 


\section{Conclusions}

As a main result, we can say that our new, $\sim 20 \mathrm{MHz}$, sub-ps Ti:S laser system supports real time, two-channel, high contrast, dual vibration resonance frequency (DVRF) CARS imaging, i.e. histology of the skin by a LSM 7 MP microscope with its original ZEN software, with properly chosen commercial bandpass and dichroic filters in front of the two NDD detectors and without any post-processing of the images like in case of our previous CARS setups used for histology [3,4]. We found that this new setup can also be used for real-time, in vivo experiments on murine skin samples, or real-time, ex vivo analysis on human pathological skin or brain tumor samples, which, in longer term, may pave the way for clinical applications during tumor surgery. Further details can be found in Ref. [7] and in corresponding oral presentation available at Biophotonics Congress 2020 web-site.

\section{Acknowledgements}

This research was funded by the National Research, Development and Innovation Fund of Hungary, contract No. K_129047 of Wigner RCP and by R\&D Ultrafast Lasers Ltd.

\section{References}

[1] D. Haluszka, K. Lőrincz, N. Kiss, R. Szipőcs, E. Kuroli, N. Gyöngyösi, N. Wikonkál, "Dietinduced obesity skin changes monitored by in vivo SHG and ex vivo CARS microscopy," Biomed. Opt. Express 7, 4480-4489 (2016). https://doi.org/10.1364/BOE.7.004480

[2] A. Duarte, C. Schnedermann, P. Kukura, "Wide-Field Detected Fourier Transf orm CARS Microscopy," Scientific Reports 6, 37516 (2016). https://doi.org/10.1038/srep37516

[3] G. Molnár, Á. Krolopp, N. Kiss, G. Tamás, R. Szipőcs, “Interferometric Spectral Modulation of sub-100-fs Pump Pulses f or High Chemical Contrast, Background Free, Real Time CARS Imaging," Biomedical Optics Congress 2018, OSA Technical Digest, paper JTh3A.29 (2018) https://doi.org/10.1364/TRANSLATIONAL.2018.JTh3A.29

[4] N. Kiss, Á. Krolopp, K. Lőrincz, A. Bánvölgyi, R. Szipőcs, and N. Wikonkál, "Stain-free Histopathology of Basal Cell Carcinoma by Dual Vibration Resonance Frequency CARS Microscopy," Pathol. Oncol. Res. 24, 927-930 (2018). https://doi.org/10.1007/s12253-017-0356-6

[5] A. Ozsvár, R. Szipőcs, Z. Ozsvár, J. Baka, P. Barzó, G. Tamás, and G. Molnár, “Quantitative analysis of lipid debris accumulation caused by cuprizone induced myelin degradation in different cns areas," Brain Research Bulletin 137, 277-284 (2018).

https://doi.org/10.1016/j.brainresbull.2018.01.003

[6] P. Antal, R. Szipőcs, "Tuneable, low-repetition-rate, cost-efficient femtosecond Ti:sapphire laser for nonlinear microscopy," Appl. Phys. B107, 17-22 (2012).

https://doi.org/10.1007/s00340-011-4830-7

[7] L. Fésűs, Á. Krolopp, G. Molnár, N. Kiss, G. Tamás, and R. Szipőcs, "A 20 MHz, sub ps, Tunable Ti:sapphire Laser System for Real Time, Stain Free, High Contrast Histology of the Skin," in Biophotonics Congress: Biomedical Optics 2020 (Translational, Microscopy, OCT, OTS, BRAIN), OSA Technical Digest (Optical Society of America, 2020), paper MTh3A.4. (2020). https://doi.org/10.1364/MICROSCOPY.2020.MTh3A.4 